\title{
A previously unknown cyclic alkanolamine and molecular ranking using the pair distribution function
}

Gianpiero Gallo*[a,b], Maxwell W. Terban*[a], Igor Moudrakovski ${ }^{[a]}$, Tatjana Huber ${ }^{[c]}$, Martin Etter $^{[\mathrm{d}]}$, Martin Ernst ${ }^{[\mathrm{c}]}$, Bernd Hinrichsen ${ }^{[\mathrm{c}]}$, Robert E. Dinnebier ${ }^{[\mathrm{a}]}$

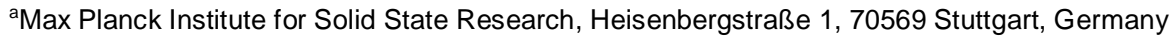

bDepartment of Chemistry and Biology "A. Zambelli”, University of Salerno, Via Giovanni Paolo II, 132, Fisciano (SA), 84084 , Italy. 'BASF SE, Carl-Bosch-Strasse 38, 67056 Ludwigshafen am Rhein, Germany.

'Deutsches Elektronen-Synchrotron (DESY), Notkestrasse 85, 22607 Hamburg, Germany.

\section{Additional tables and figures}

\section{Contents}

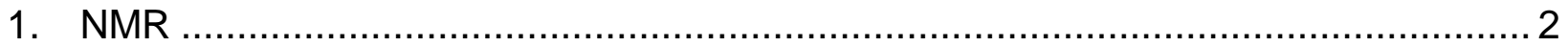

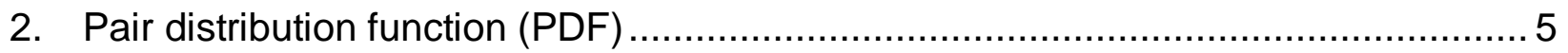

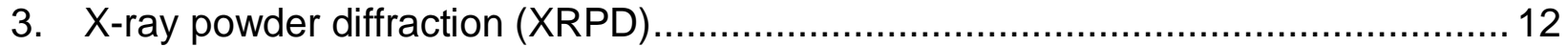

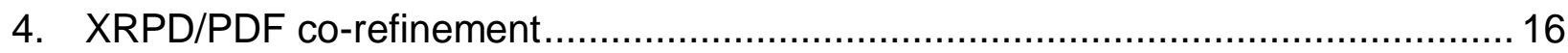

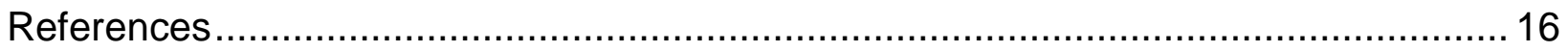




\section{NMR}

${ }^{1} \mathrm{H}$ NMR, solution in $\mathrm{D}_{2} \mathrm{O}$

兽

H-O $\mathrm{HO}-\mathrm{CH}_{2}-(\mathrm{CH}<)$

$-\left(\mathrm{CH}_{2}\right)-\underline{\mathrm{CH}}<$

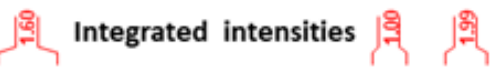

Residual $\mathrm{H}$ in $\mathrm{D}_{2} \mathrm{O}$ adds up to HO- signal

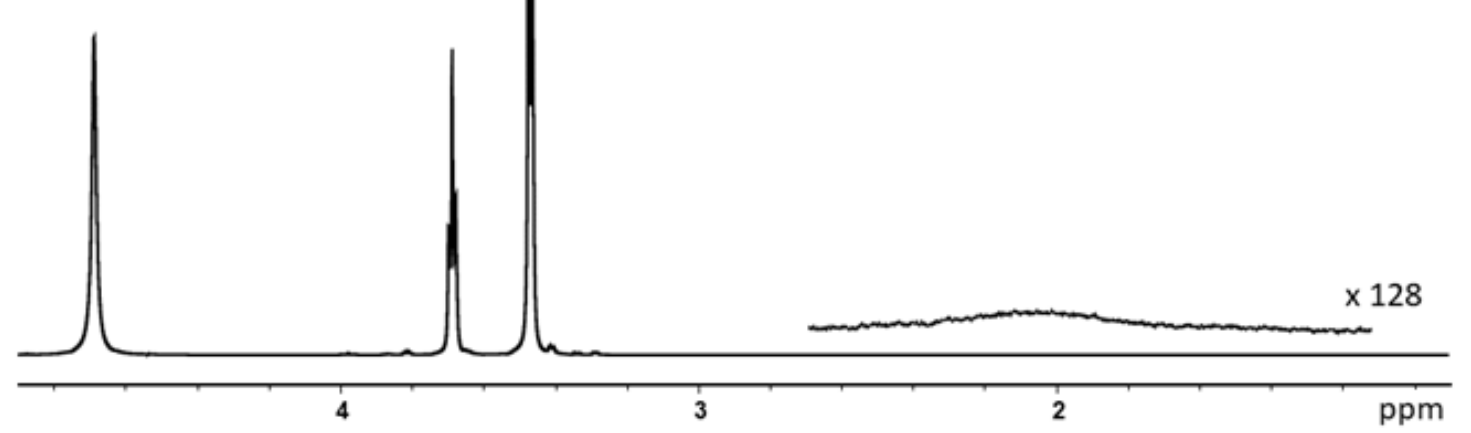

Figure S1. ${ }^{1} \mathrm{H}$ NMR spectrum of a solution of $\mathbf{1}$ in $\mathrm{D}_{2} \mathrm{O}$.

${ }^{13} \mathrm{C}$ NMR, solution in $\mathrm{D}_{2} \mathrm{O}$

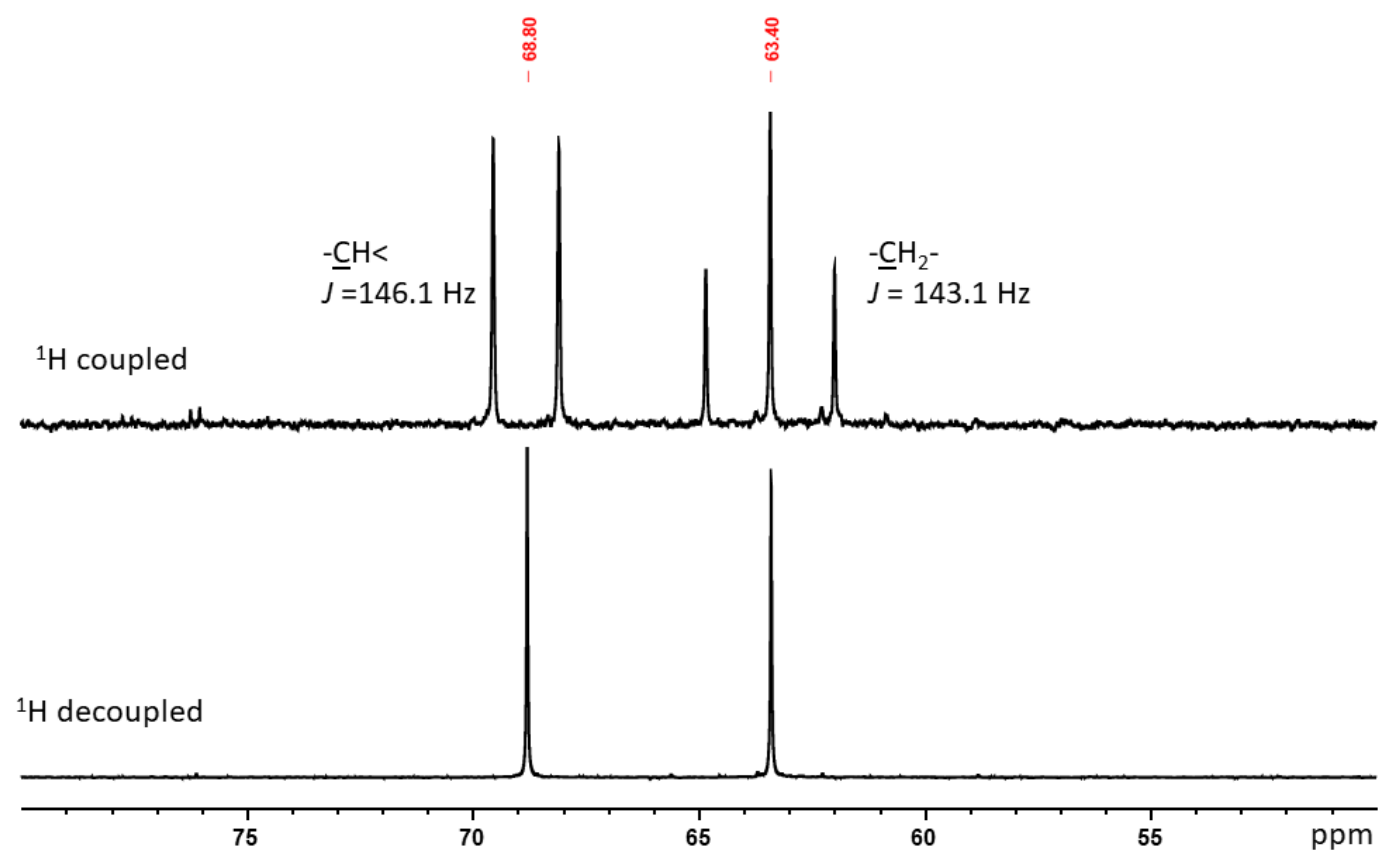

Figure S2. ${ }^{13} \mathrm{C}$ NMR spectra $\left({ }^{1} \mathrm{H}\right.$ coupled, above and ${ }^{1} \mathrm{H}$ decoupled, below) of a solution of $1 \mathrm{in}$ DMSO-d6. 
${ }^{15} \mathrm{~N}$ DEPT NMR, solution in $\mathrm{D}_{2} \mathrm{O}$

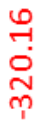

$-\mathrm{CH}_{\mathrm{m}}-\underline{\mathrm{N}} \mathrm{H}_{\mathrm{n}}-$

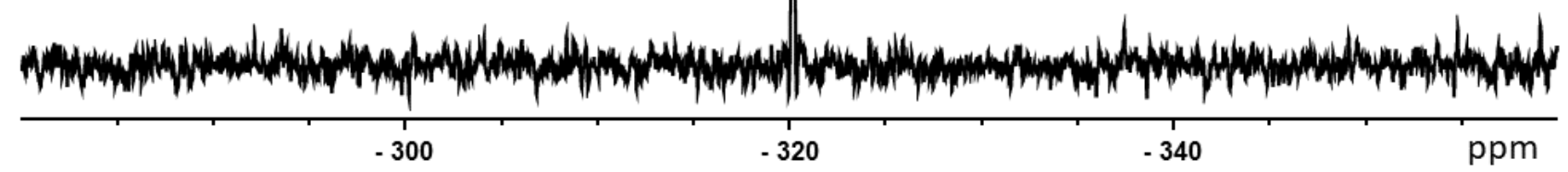

Figure S3. ${ }^{15} \mathrm{~N}$ NMR spectrum of a solution of 1 in DMSO-d6.

${ }^{1} \mathrm{H}$ NMR, solution in DMSO-d6

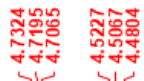

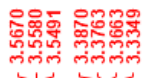

$\mathrm{HO}-\mathrm{CH}_{2}-(\mathrm{CH}<)$

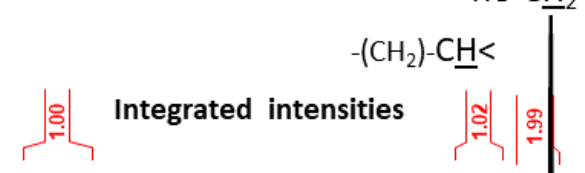

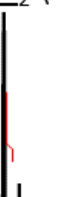

$\underline{\mathrm{H}}-\mathrm{O}\left(\mathrm{CH}_{2}\right)$

Residual $\mathrm{H}$ in

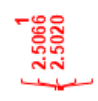

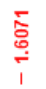

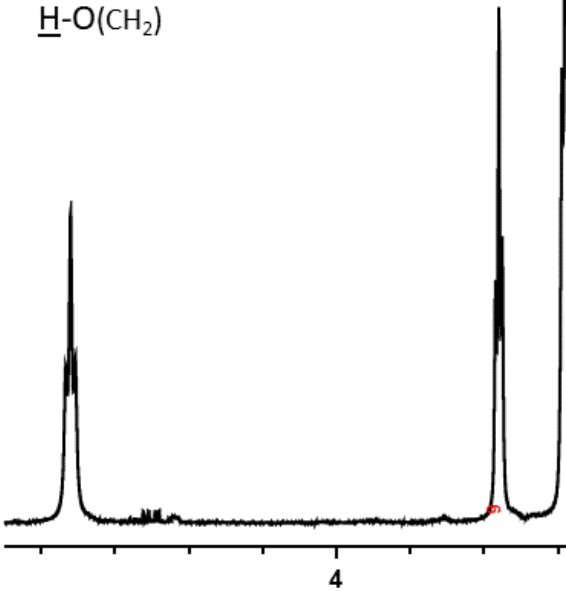

$\mathrm{H}_{2} \mathrm{O} / \mathrm{DHO}$

DMSO-d6

in DMSO-d6

DMO-d6

$-\mathrm{NH}-$

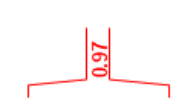




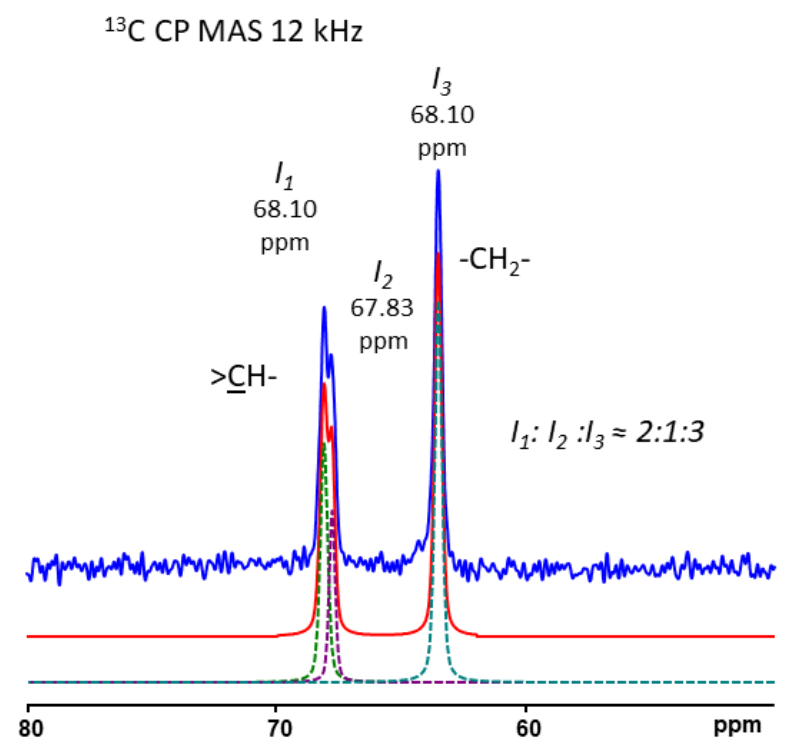

${ }^{15} \mathrm{~N}$ CP MAS $5 \mathrm{kHz}$

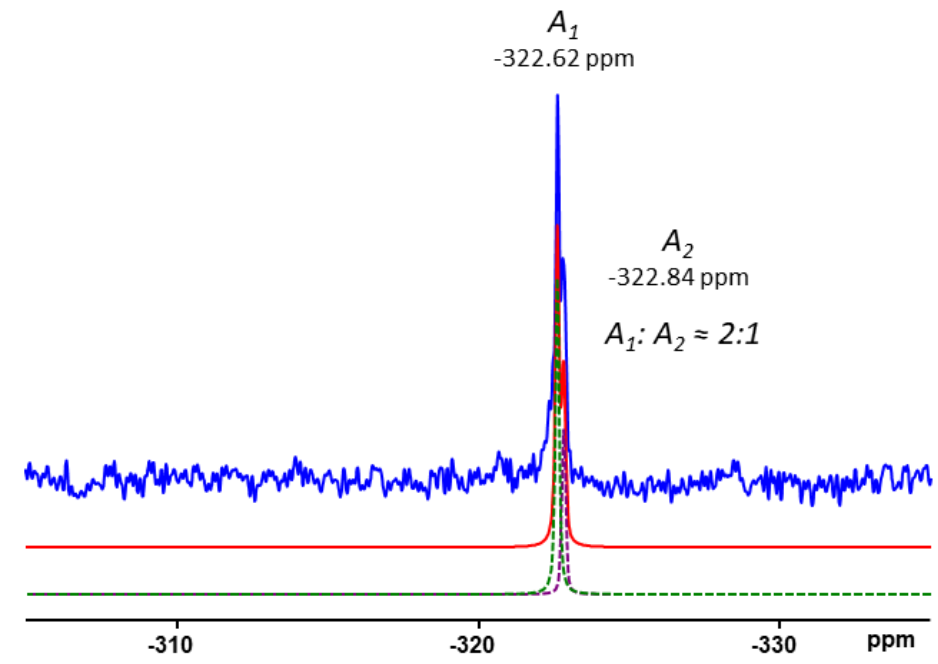

Figure S5. ${ }^{13} \mathrm{C}$ (right) and ${ }^{15} \mathrm{~N}$ (left) CP MAS solid-state NMR spectra of 1. 


\section{Pair distribution function (PDF)}

Total scattering measurements over a wide range of momentum transfer and with good statistics are required to obtain suitable PDFs for structure analysis. The coherent powder diffraction intensities $I_{c}(Q)$ are normalized by the form factors of the sample composition to obtain the total scattering structure function $S(Q)$ by

$$
S(Q)=\frac{I_{c}(Q)-\left\langle f(Q)^{2}\right\rangle+\langle f(Q)\rangle^{2}}{\langle f(Q)\rangle^{2}},
$$

which is then Fourier transformed by

$$
G(r)=\frac{2}{\pi} \int_{Q_{\min }}^{Q_{\max }} Q[S(Q)-1] \sin (Q r) d Q .
$$

$F(Q)=Q[S(Q)-1]$ is the reduced total scattering structure function, and $G(r)$ is the PDF. In practice, values of $Q_{\min }$ and $Q_{\max }$ are determined by the experimental setup, and $Q_{\max }$ is often reduced below the experimental maximum to reduce the effects of low signal-to-noise in the high$Q$ region on the Fourier transformation. The PDF gives the scaled probability of finding two atoms in a material a distance $r$ apart and is relative to the density of atom pairs in the material. $G(r)$ can be calculated from a known structure model according to

$$
G(r)=\frac{1}{\mathrm{r} N}\left(\sum_{i} \sum_{j \neq i} \frac{f_{i} f_{j}}{\langle f\rangle^{2}} \delta\left(r-r_{i j}\right)\right)-4 \pi r \rho_{0} .
$$

Here, $\rho_{0}$ is the average number density of the material, and the sums run over all atoms in the sample; $f_{i}$ is the scattering factor of atom $i,\langle f\rangle$ is the average scattering factor, and $r_{i j}$ is the distance between atoms $i$ and $j$. Refinement and fitting of crystallographic structures to the PDF data were performed using TOPAS $v 6^{1}$.

$F(Q)$ for discrete molecules was calculated using the Debye function as implemented in Diffpy$\mathrm{CMI}^{2}$, wherein

$$
F(Q)=\frac{1}{N} \sum_{i} \sum_{j \neq i} \frac{f_{i} f_{j}}{\langle f\rangle^{2}} \frac{\sin (Q r)}{r} .
$$

The calculations were performed with the atomic displacement parameter $B_{\text {iso }}=0.2 \AA^{2}$ for every atom, $Q_{\text {damp }}=0.03 \AA^{-1}$ and $Q_{\text {broad }}=0.01 \AA^{-1}$. $G(r)$ was subsequently obtained by inputting this into Eq. 2 with $Q_{\min }=1.5 \AA^{-1}$ and $Q_{\max }=22 \AA^{-1}$. For comparison and ranking, the Pearson product-momentum correlation coefficient (PCC) is calculated by 


$$
\mathrm{PCC}=\frac{1}{n-1} \sum_{i=0}^{n}\left(\frac{X_{i}-\bar{X}}{\sigma_{x}}\right)\left(\frac{Y_{i}-\bar{Y}}{\sigma_{y}}\right)
$$

Where $\bar{X}$ and $\bar{Y}$ are the means and $\sigma_{x}$ and $\sigma_{y}$ are the standard deviations of the respective datasets. This calculation gives a value between -1 and 1 where -1 implies anticorrelation, 0 implies no correlation, and 1 implies perfect correlation.

(a)

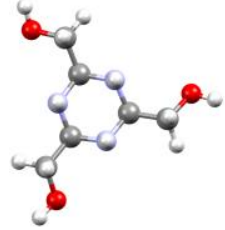

(b)

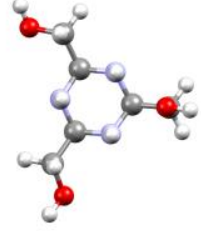

(c)

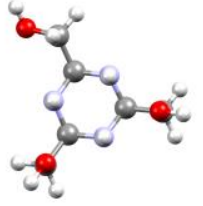

(d)

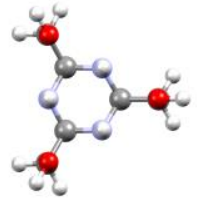

all hydroxymethyl groups twisted down, near plane of ring
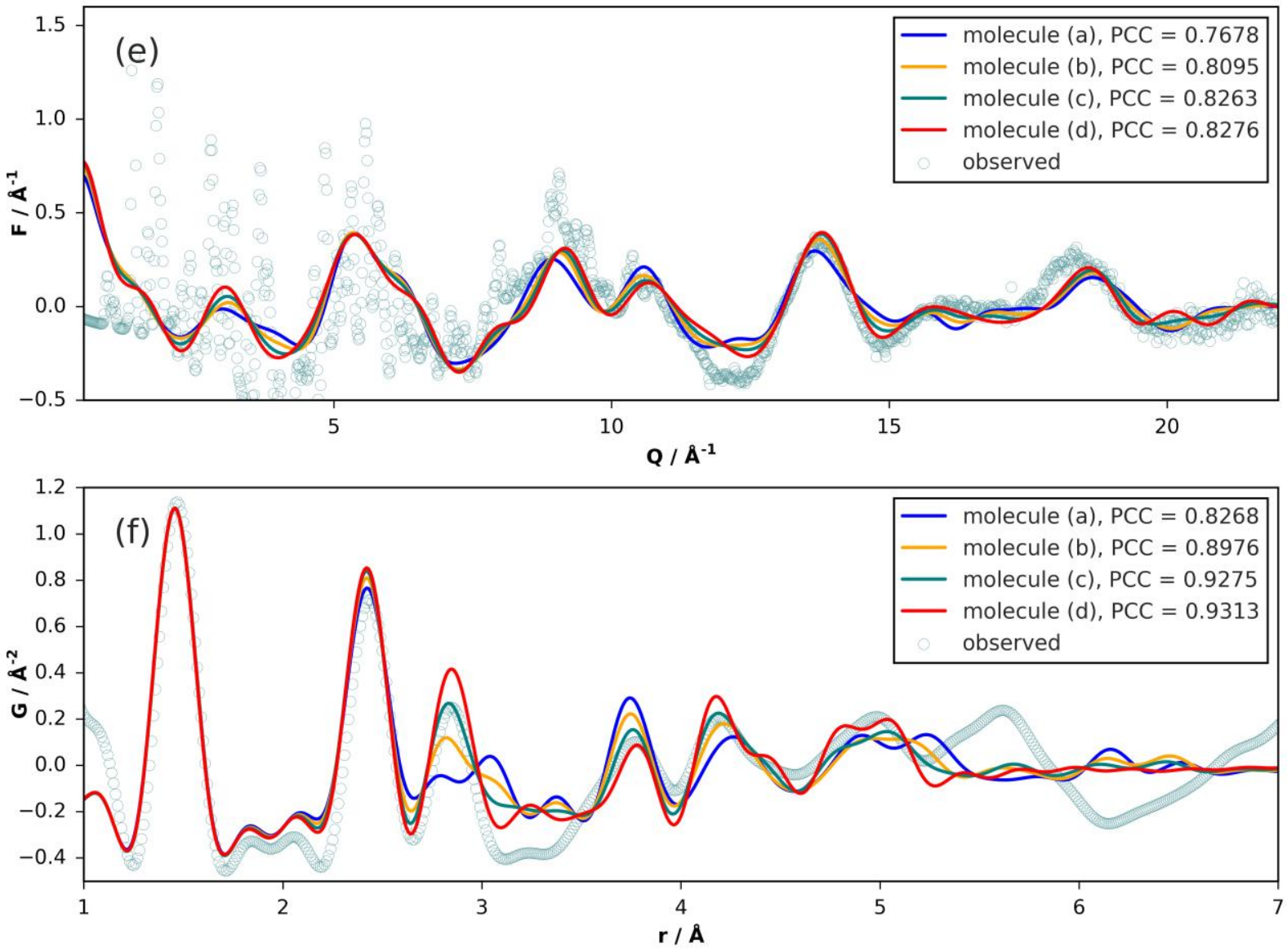

Figure S6. Four representative conformations of the best molecular structure investigated (a-d) looking along the plane of the ring. Comparisons of simulated and experimental $F(Q)$ and $G(r)$ and PCC values (compared over ranges of $Q=6.0-22.0 \AA^{-1}$ and $r=1.9-5.25 \AA$ ) are given in (e) and (f) respectively. Conformation (a) has all hydroxymethyl groups twisted down, pointing close to but just out of the plane of the ring. Molecules (b-d) represent conformations with one, two, or all three hydroxymethyl groups flipped upward, pointing perpendicular to the plane of the ring, respectively. 


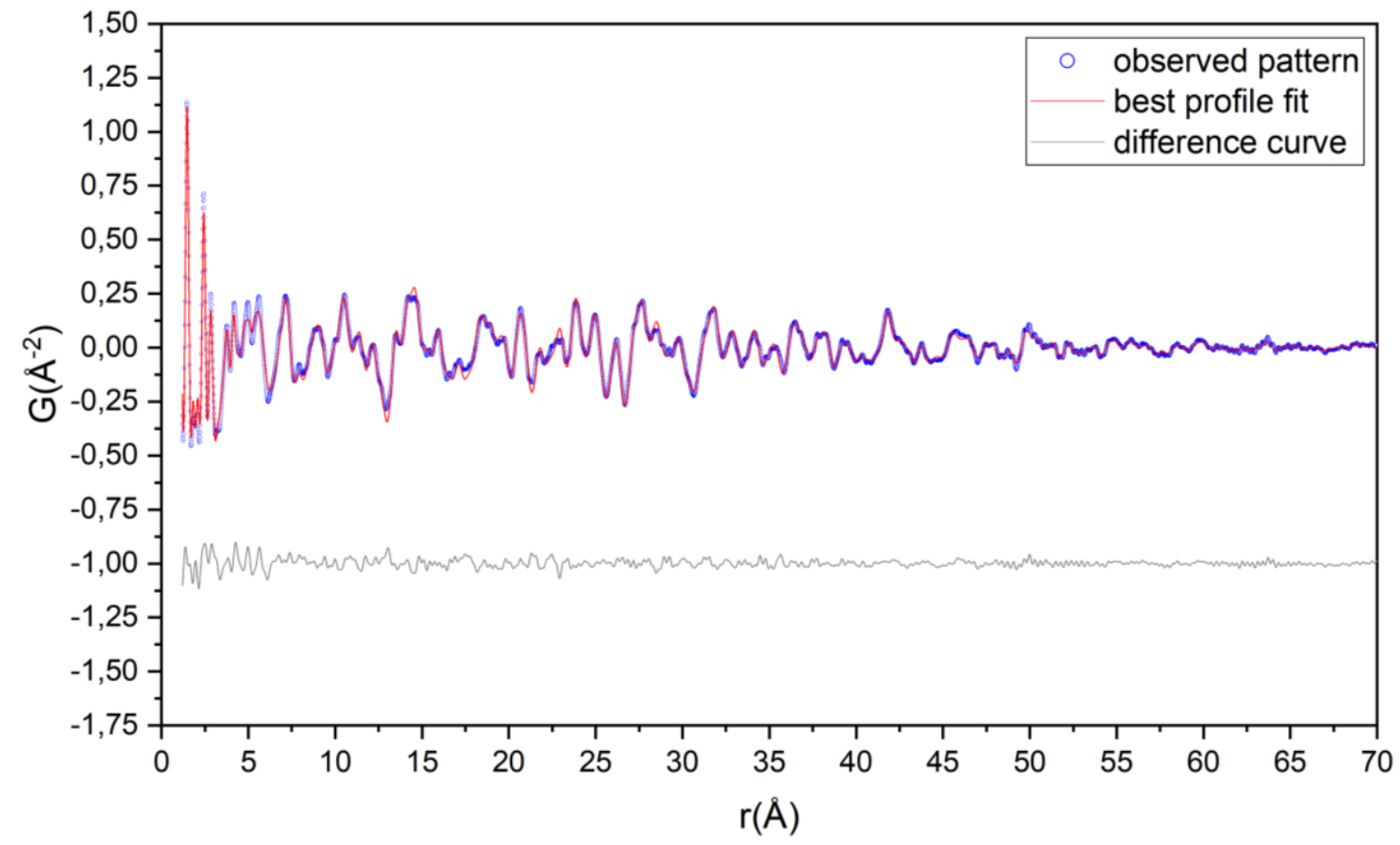

Figure S7. PDF fit of the model with Ama2 space group (model 1 ) in a ' $r$ '-range from 1.2 to $70 \AA$. The blue circles and red solid line correspond to measured and simulated PDFs, respectively. The grey solid line offset below denotes the difference curve.

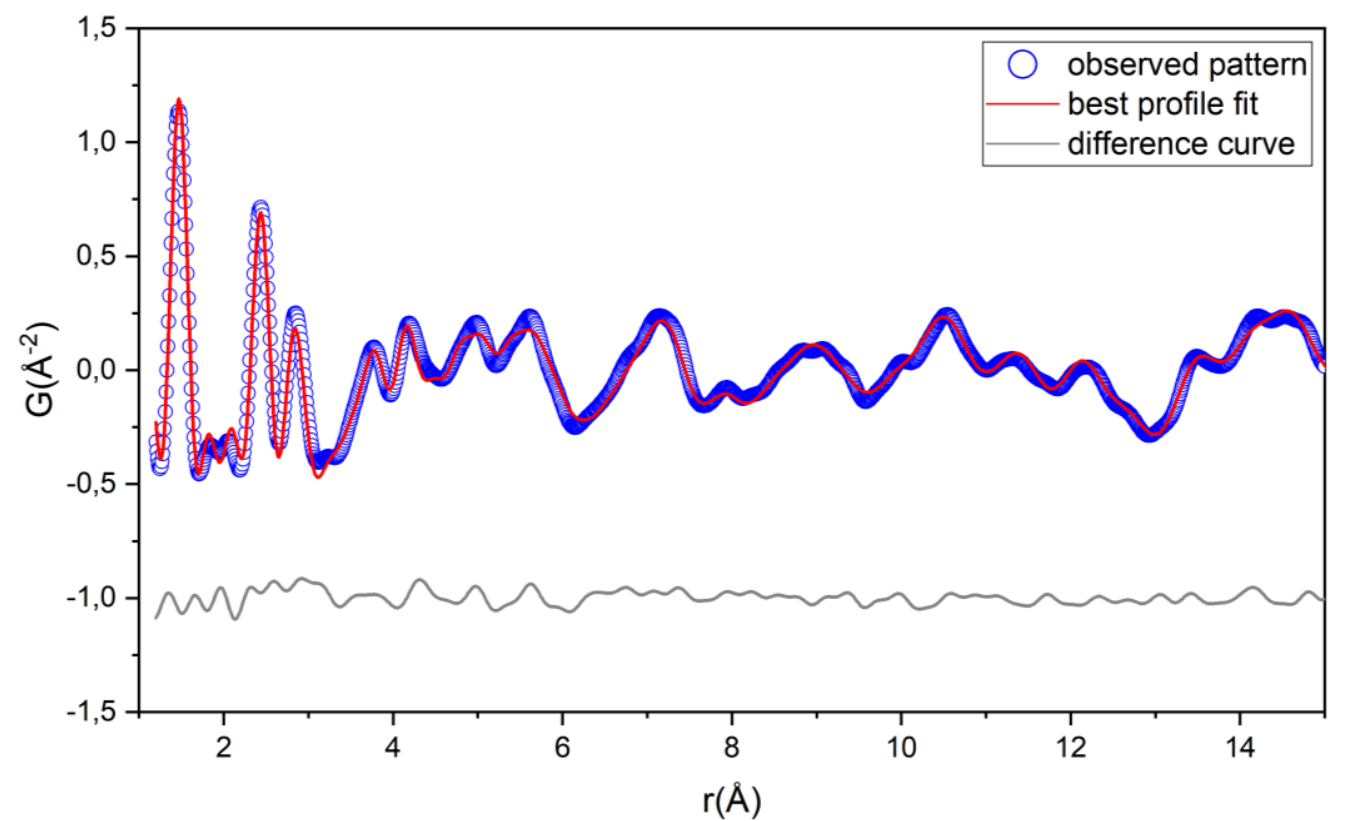

Figure S8. PDF fit of the model with Ama2 space group (model 1) in a ' $r$ '-range from 1.2 to $15 \AA$. The blue circles and red solid line correspond to measured and simulated PDFs, respectively. The grey solid line offset below denotes the difference curve. 


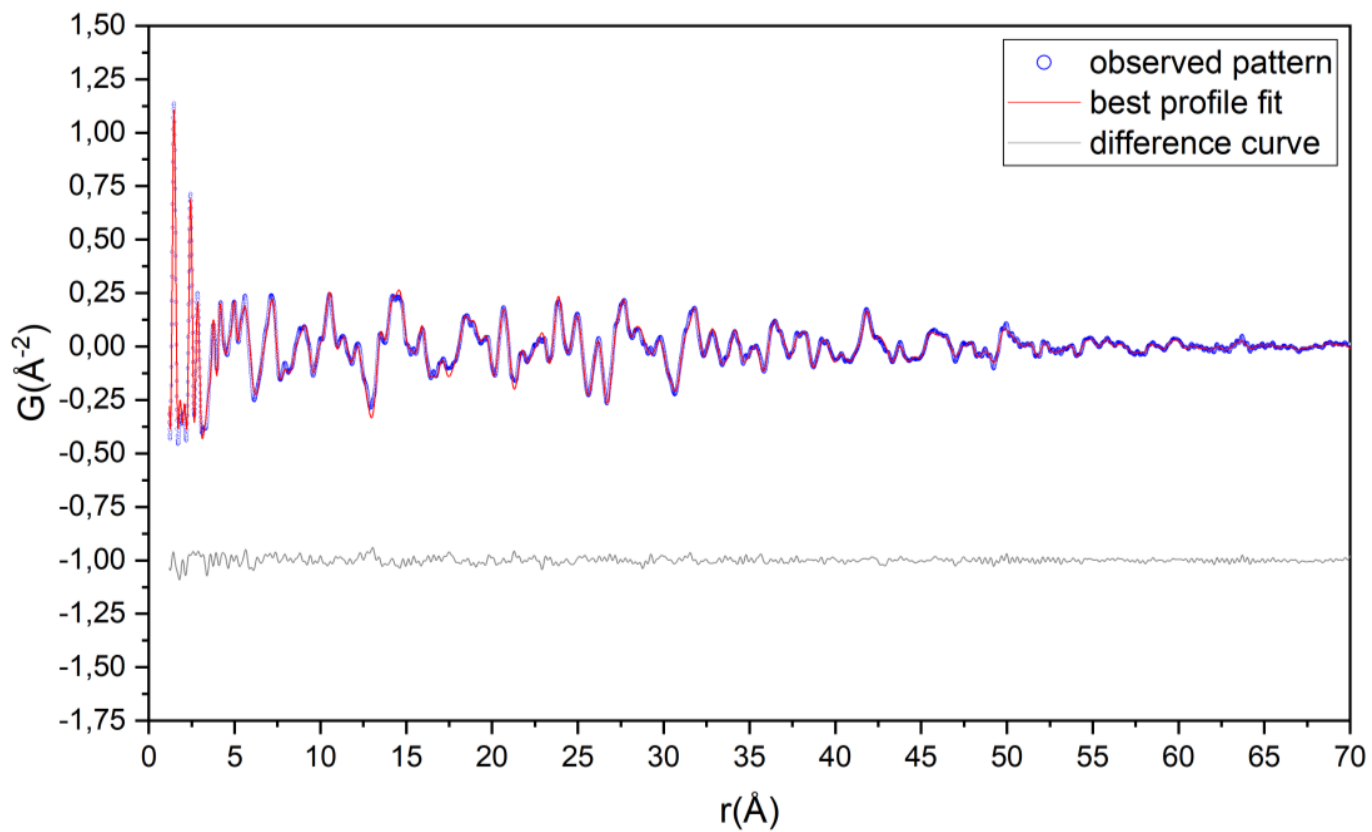

Figure S9. PDF fit of the model with $C 2$ space group (model 2) in a ' $r$-range from 1.2 to $70 \AA$. The blue circles and red solid line correspond to measured and simulated PDFs, respectively. The grey solid line offset below denotes the difference curve.

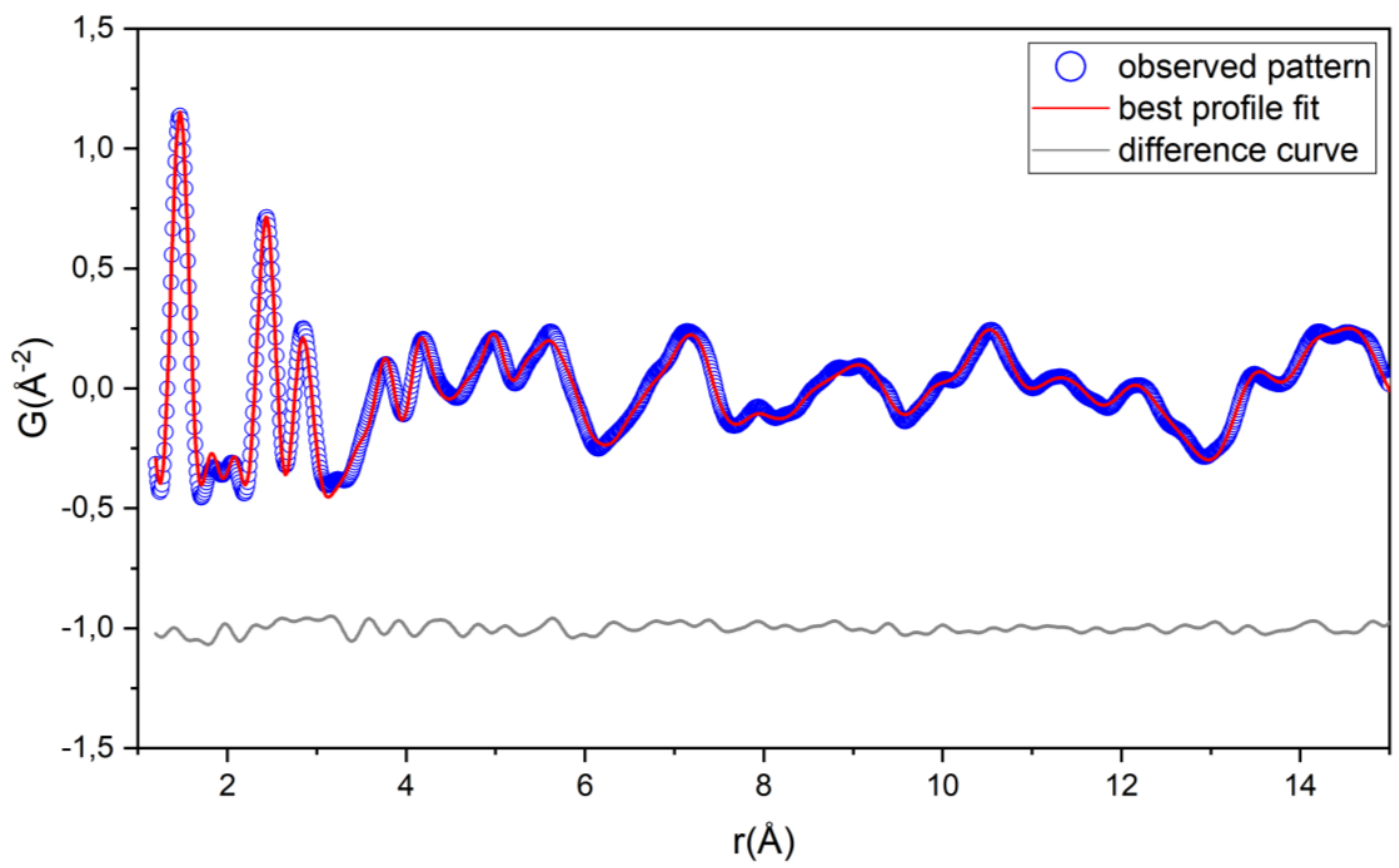

Figure S10. PDF fit of the model with $C 2$ space group (model 2) in a ' $r$ '-range from 1.2 to $15 \AA$. The blue circles and red solid line correspond to measured and simulated PDFs, respectively. The grey solid line offset below denotes the difference curve. 


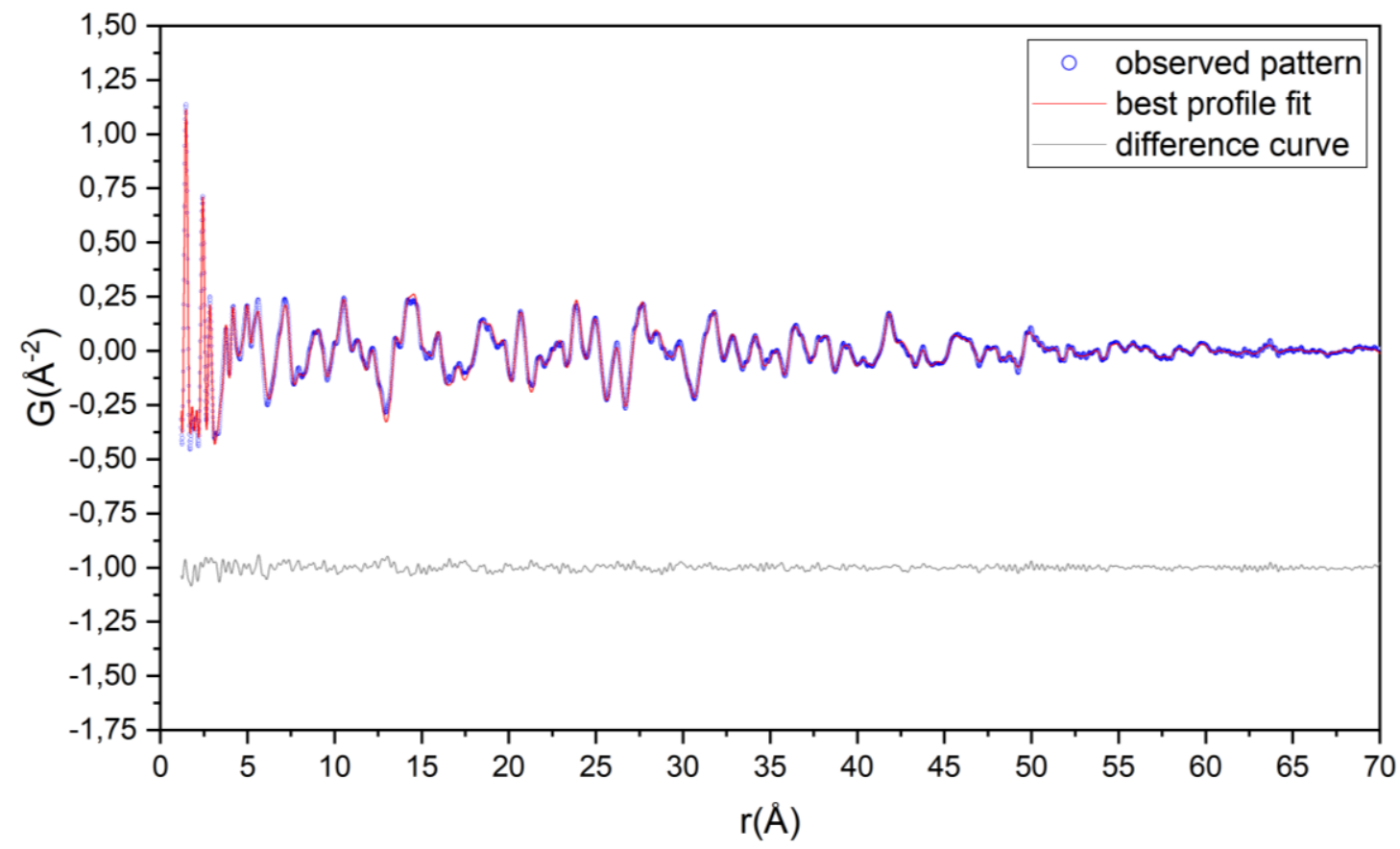

Figure S11. PDF fit of the model with $P 1$ space group (model 3 ) in a ' $r$ 'range from 1.2 to $70 \AA$. The blue circles and red solid line correspond to measured and simulated PDFs, respectively. The grey solid line offset below denotes the difference curve.

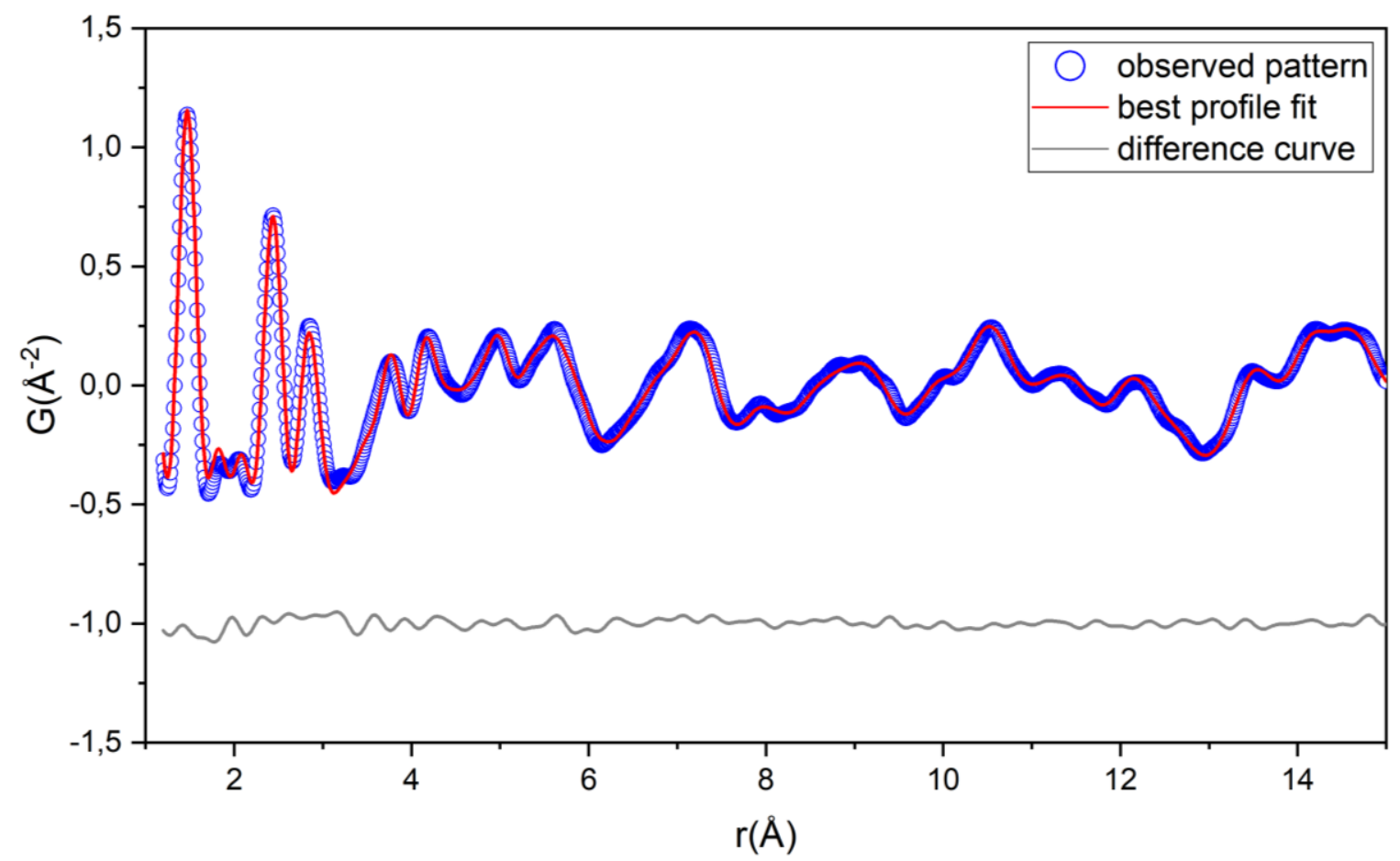

Figure S12. PDF fit of the model with $P 1$ space group (model 3 ) in a ' $r$ '-range from 1.2 to $15 \AA$. The blue circles and red solid line correspond to measured and simulated PDFs, respectively. The grey solid line offset below denotes the difference curve. 


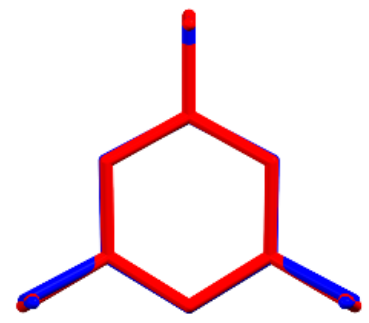

(a)

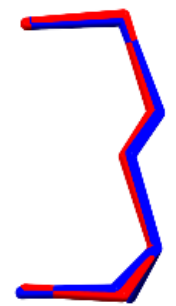

(b)

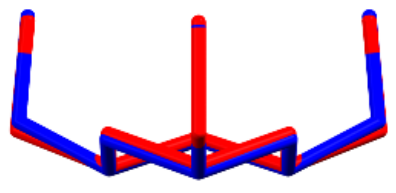

(c)

Figure S13. Superimposition of the molecules obtained from XRPD (red) and from PDF using the model Ama2 (blue). Root-mean-square deviation (RMSD): 0.738.

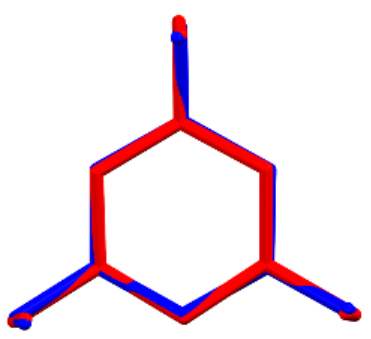

(a)

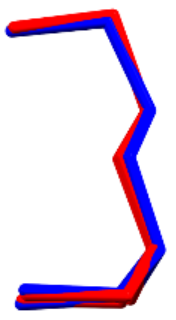

(b)

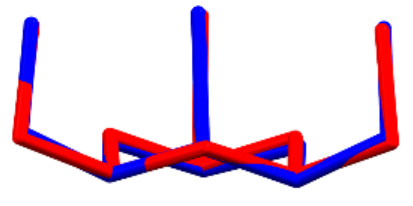

(c)

Figure S14. Superimposition of the molecules obtained from XRPD (red) and from PDF using the model C2 (blue). RMSD: 0.805.

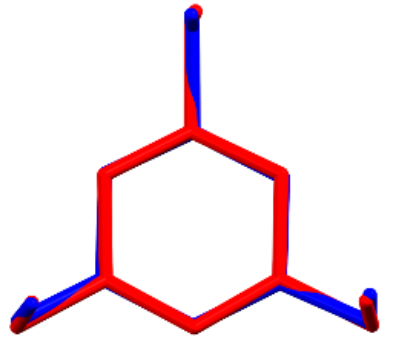

(a)

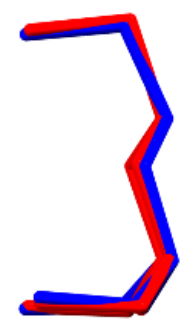

(b)

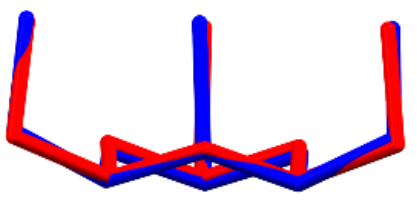

(c)

Figure S15. Superimposition of the molecules obtained from XRPD (red) and from PDF using the model $P 1$ (blue). RMSD: 0.868. 


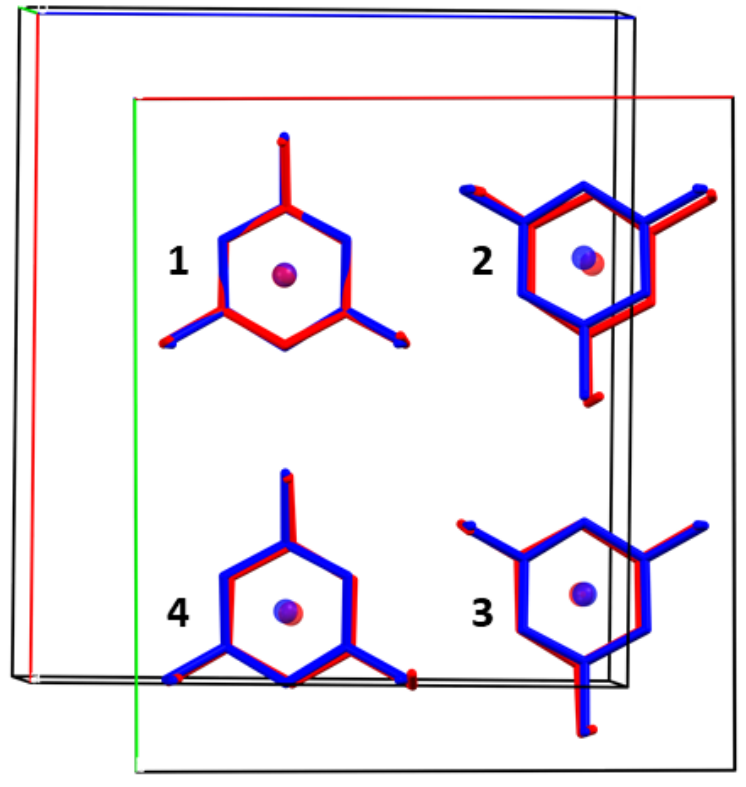

(a)

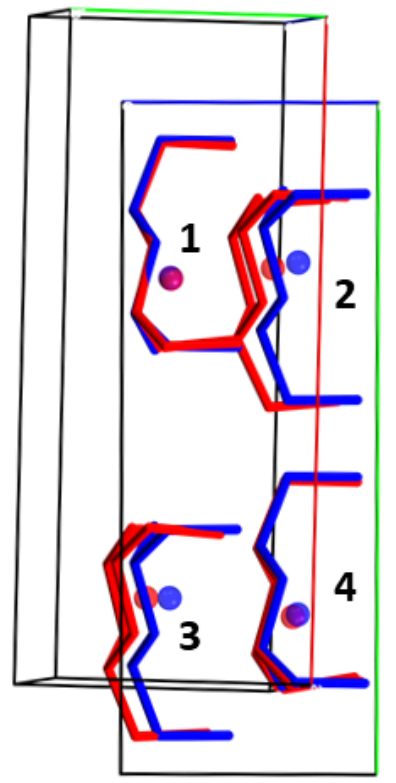

(b)

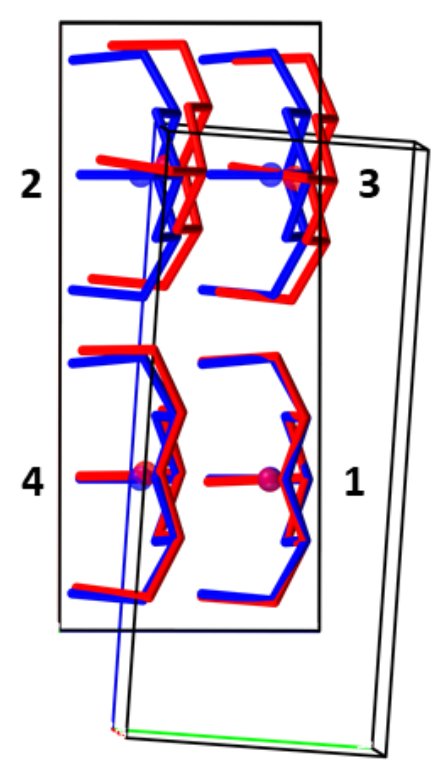

(c)

Figure S16. Comparison between the unit cells contents obtained from PDF refinements of the structure models with space groups $A m a 2$ (blue) and $P 1$ (red) in the range 1.2-15 $\mathrm{A}$. Non-H atoms of the molecules 1 were superimposed in order to show the difference between the two models. Distances between centroids, calculated using all non-H atoms, are $0.555 \AA$ in $2,0.482 \AA$ in 3 , and $0.149 \AA$ in 4 . Centroids related to the models with space groups $A m a 2$ and $P 1$ are depicted in blue and red, respectively. Hydrogen atoms have been omitted for clarity.

Table S1. Crystallographic and PDF Refinement data of 1.

\begin{tabular}{|c|c|c|c|c|c|c|}
\hline Compound & \multicolumn{2}{|c|}{ Model 1} & \multicolumn{2}{|c|}{ Model 2} & \multicolumn{2}{|c|}{ Model 3} \\
\hline Crystal & \multicolumn{2}{|c|}{ Orthorhombic } & \multicolumn{2}{|c|}{ Monoclinic } & \multicolumn{2}{|c|}{ Triclinic } \\
\hline Space group & \multicolumn{2}{|c|}{ Ama2 } & \multicolumn{2}{|c|}{$C 2$} & \multicolumn{2}{|c|}{$P 1$} \\
\hline$a / \AA$ & $12.1096(9)$ & $12.114(5)$ & $13.5582(8)$ & $13.567(6)$ & $13.5580(9)$ & $13.565(8)$ \\
\hline b/ $\mathbf{A}$ & $13.558(1)$ & $13.637(3)$ & $5.2091(3)$ & $5.201(2)$ & $5.2080(3)$ & $5.207(3)$ \\
\hline 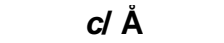 & $5.2087(4)$ & $5.197(3)$ & $12.1153(7)$ & $12.140(4)$ & $12.1126(7)$ & $12.120(6)$ \\
\hline$\alpha /{ }^{\circ}$ & 90 & 90 & 90 & 90 & 89.94(4) & $90.1(3)$ \\
\hline$\beta /{ }^{\circ}$ & 90 & 90 & $90.00(3)$ & $90.3(2)$ & $90.03(3)$ & $90.1(3)$ \\
\hline$y /{ }^{\circ}$ & 90 & 90 & 90 & 90 & $90.08(3)$ & $90.2(3)$ \\
\hline $\mathbf{V} / \dot{A}^{3}$ & $855.2(1)$ & $858.6(7)$ & $855.65(9)$ & $856.7(6)$ & $855.3(1)$ & $856.0(8)$ \\
\hline $\mathbf{T} / \mathrm{K}$ & 298 & 298 & 298 & 298 & 298 & 298 \\
\hline $\begin{array}{c}\text { refıned } \\
\text { parameters }\end{array}$ & \multicolumn{2}{|c|}{16} & \multicolumn{2}{|c|}{24} & \multicolumn{2}{|c|}{47} \\
\hline $\mathbf{R}_{w p} / \%$ [a] & 17.9 & 14.6 & 13.3 & 10.3 & 12.9 & 9.8 \\
\hline $\begin{array}{l}\text { Starting ' } r \text { - } \\
\text { range used/ } \AA\end{array}$ & 1.2 & 1.2 & 1.2 & 1.2 & 1.2 & 1.2 \\
\hline $\begin{array}{c}\text { Final ' } r \text { '- } \\
\text { range used/ } \\
\underset{\AA}{A}\end{array}$ & 70 & 15 & 70 & 15 & 70 & 15 \\
\hline
\end{tabular}

[a] as defined in TOPAS 4. 


\section{X-ray powder diffraction (XRPD)}

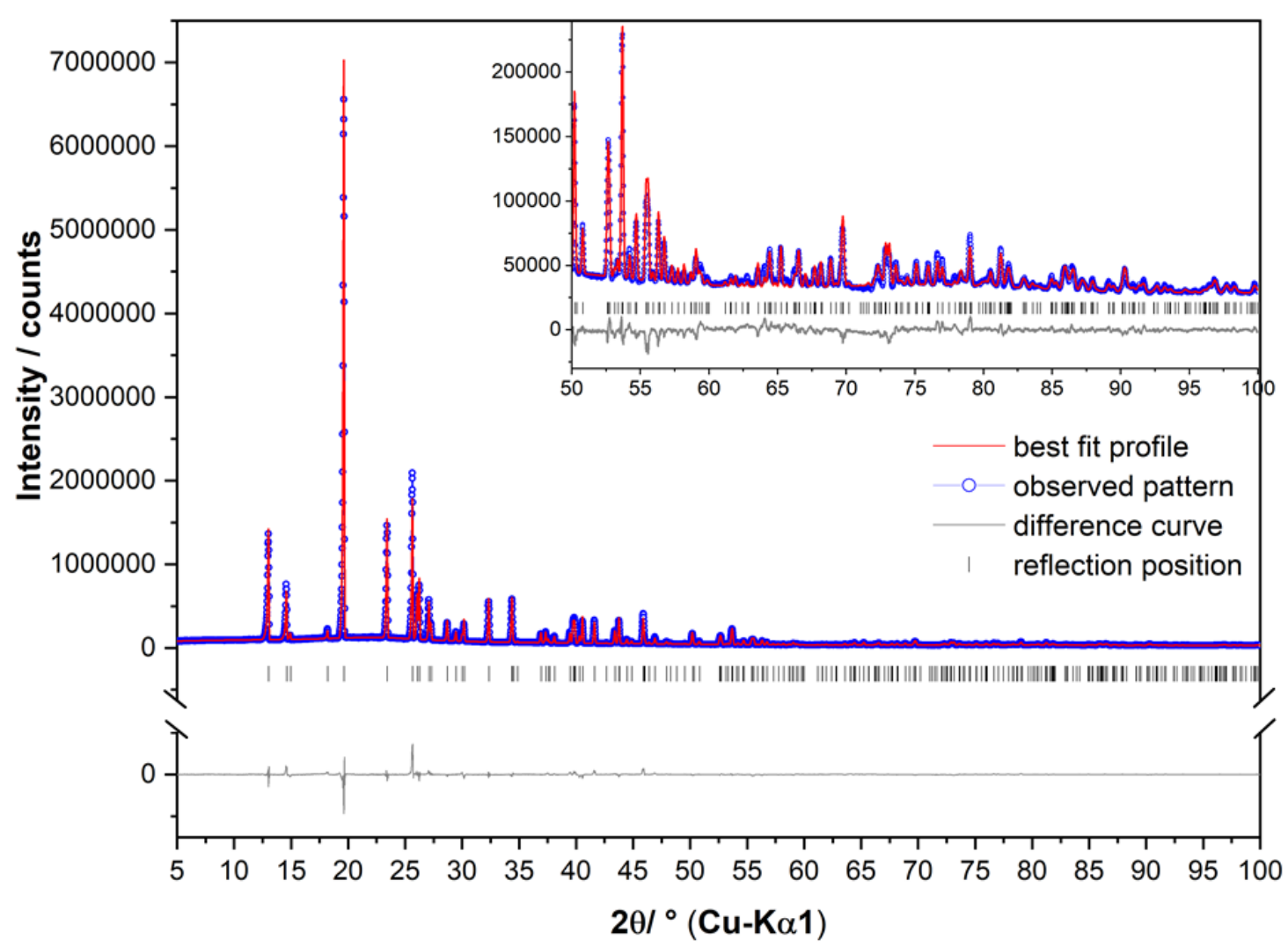

Figure S17. Rietveld plot of the model with Ama2 space group obtained from PDF refinement. The observed pattern (circles), the best Rietveld fit profile (line) and the difference curve between the observed and the calculated profiles (below) are shown. The high angle part starting at $50^{\circ}$ in $2 \theta$ is enlarged for clarity. 


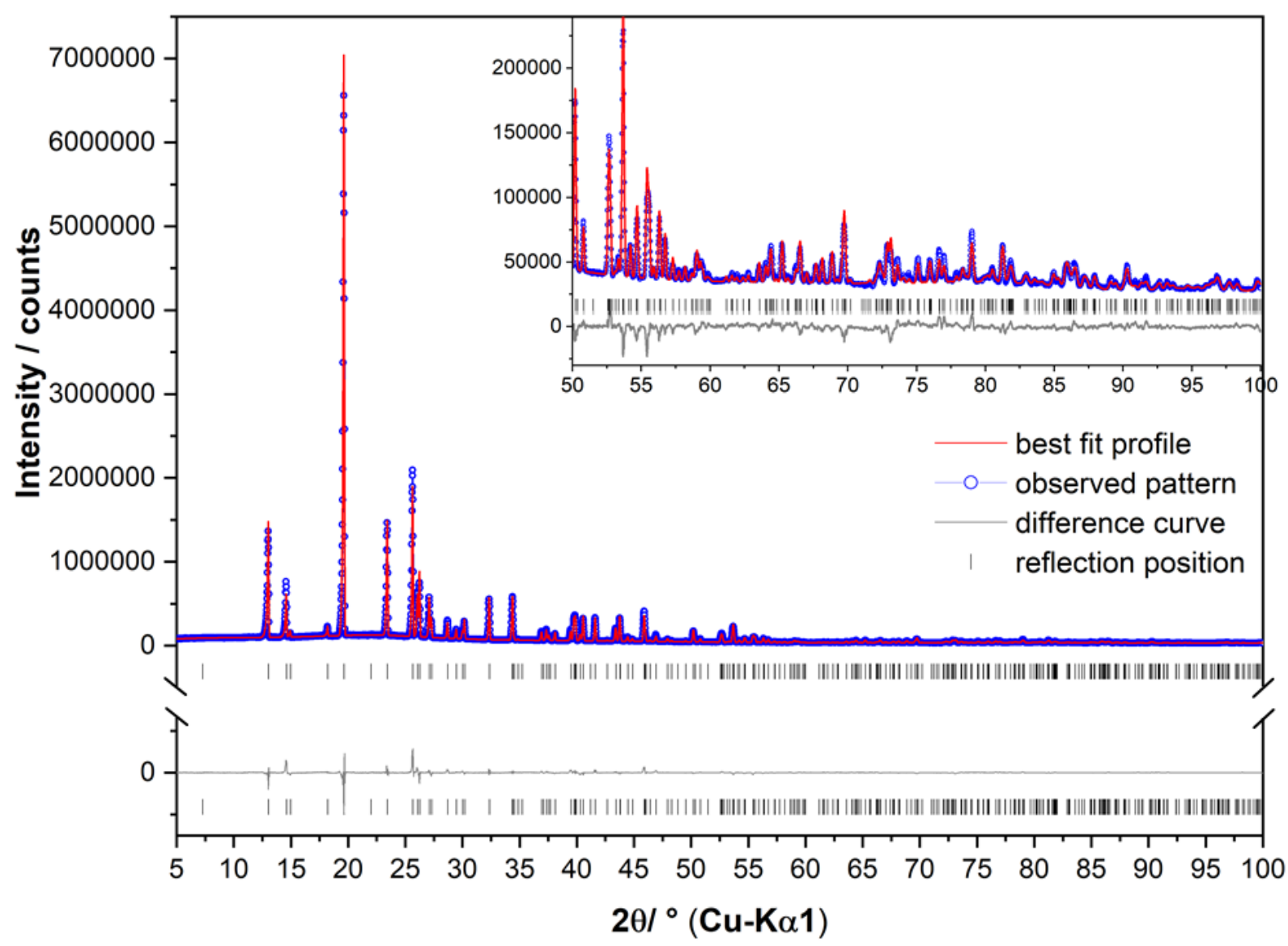

Figure S18. Rietveld plot of the model with $C 2$ space group obtained from PDF refinement. The observed pattern (circles), the best Rietveld fit profile (line) and the difference curve between the observed and the calculated profiles (below) are shown. The high angle part starting at $50^{\circ}$ in $2 \theta$ is enlarged for clarity. 


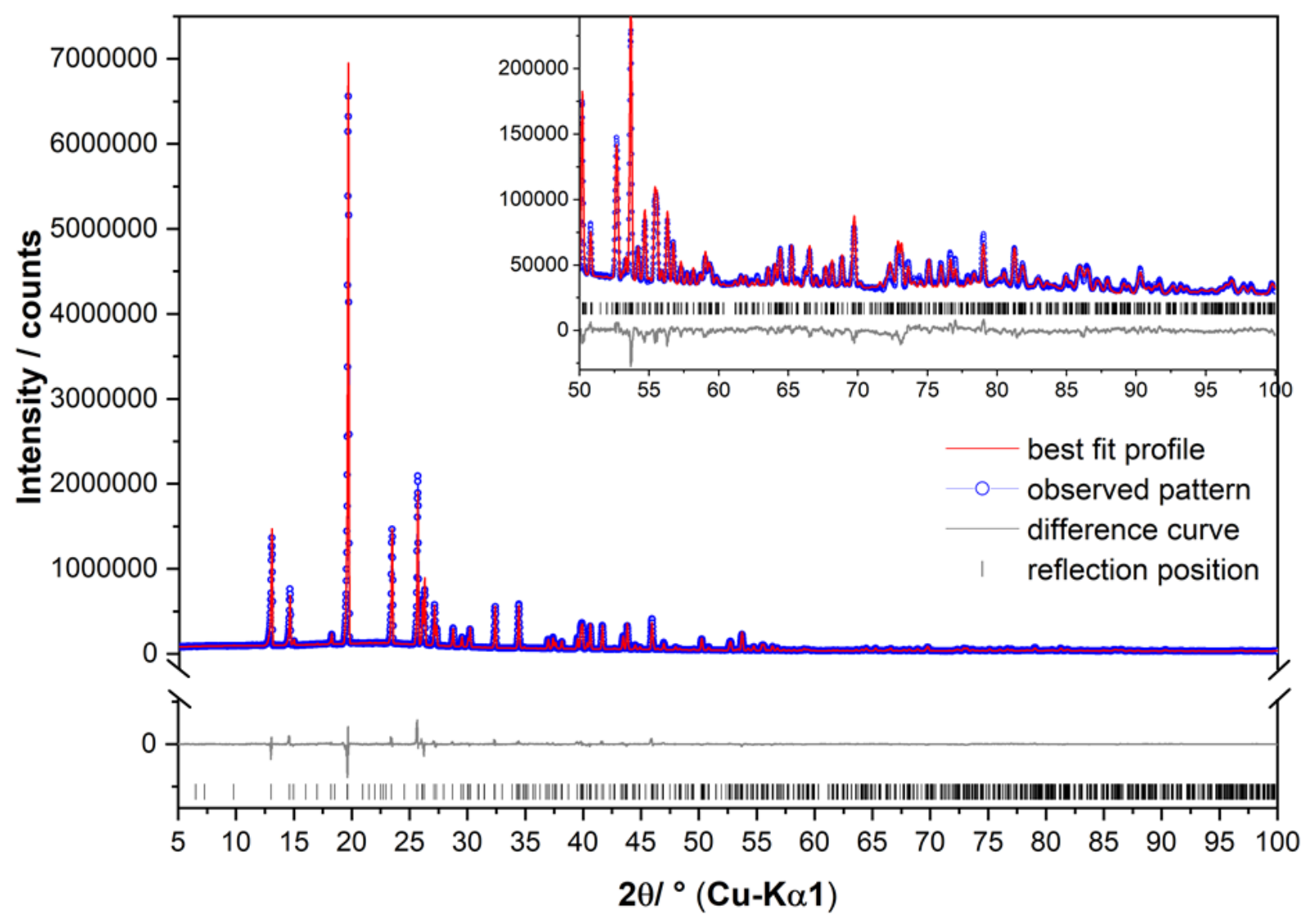

Figure S19. Rietveld plot of the model with $P 1$ space group obtained from PDF refinement. The observed pattern (circles), the best Rietveld fit profile (line) and the difference curve between the observed and the calculated profiles (below) are shown. The high angle part starting at $50^{\circ}$ in $2 \theta$ is enlarged for clarity.

Table S2. Crystallographic and Rietveld Refinement data of 1 using the models obtained from PDF refinements.

\begin{tabular}{|c|c|c|c|}
\hline Compound & Model 1 & Model 2 & Model 3 \\
\hline Crystal system & Orthorhombic & Monoclinic & Triclinic \\
\hline Space group & Ama2 & $C 2$ & $P 1$ \\
\hline Wavelength / A & 1.5406 & 1.5406 & 1.5406 \\
\hline$a / \AA$ & $13.5543(2)$ & $13.5550(2)$ & $13.5545(2)$ \\
\hline $\boldsymbol{b} / \ddot{A}$ & $5.20768(8)$ & $5.20795(7)$ & $5.20783(6)$ \\
\hline$c / \check{A}$ & $12.1059(2)$ & 12.1064(2) & $12.1061(1)$ \\
\hline $\mathbf{V} / \mathbf{A}^{3}$ & $854.52(2)$ & $854.64(2)$ & $854.56(2)$ \\
\hline $\mathbf{R}_{w p} / \%$ [a] & 6.35 & 6.08 & 5.53 \\
\hline $\mathbf{R}_{p} / \%$ [a] & 5.54 & 4.32 & 4.11 \\
\hline $\mathbf{R}_{\text {Bragg }} / \%$ [a] & 5.26 & 5.14 & 4.67 \\
\hline $\begin{array}{l}\text { Starting angle } \\
\text { measured } /{ }^{\circ} 2 \theta\end{array}$ & 0 & 0 & 0 \\
\hline $\begin{array}{c}\text { Final angle } \\
\text { measured } /{ }^{\circ} 2 \theta\end{array}$ & 110 & 110 & 110 \\
\hline $\begin{array}{l}\text { Starting angle used / } \\
{ }^{\circ} 2 \theta\end{array}$ & 5 & 5 & 5 \\
\hline $\begin{array}{c}\text { Final angle used } /{ }^{\circ} \\
2 \theta\end{array}$ & 100 & 100 & 100 \\
\hline Step width / ${ }^{\circ} 2 \theta$ & 0.01 & 0.01 & 0.01 \\
\hline
\end{tabular}


Table S3. Atomic coordinates of 1.

\begin{tabular}{cccccccc}
\hline Atom & Wyck. & $\begin{array}{c}\text { Site } \\
\text { symmetry }\end{array}$ & S.O.F. & $\boldsymbol{x} / \mathbf{a}$ & $\boldsymbol{y} / \mathbf{b}$ & $\boldsymbol{z} / \mathbf{c}$ & ${\mathbf{B} / \mathbf{A}^{2}}$ \\
\hline C1 & $4 b$ & $\mathrm{~m} .$. & 1 & $1 / 4$ & $0.16077(4)$ & $0.00302(15)$ & $2.05(4)$ \\
C2 & $8 c$ & 1 & 1 & $0.34991(8)$ & $0.31422(12)$ & $0.0053(5)$ & $2.05(4)$ \\
N1 & $8 c$ & 1 & 1 & $0.35158(9)$ & $0.21088(4)$ & $-0.0820(4)$ & $2.05(4)$ \\
N2 & $4 b$ & $m .$. & 1 & $1 / 4$ & $0.36918(16)$ & $-0.0695(5)$ & $2.05(4)$ \\
C3 & $4 b$ & $\mathrm{~m} .$. & 1 & $1 / 4$ & $0.05301(0)$ & $-0.0832(5)$ & $2.05(4)$ \\
C4 & $8 c$ & 1 & 1 & $0.45271(8)$ & $0.36798(12)$ & $-0.0917(8)$ & $2.05(4)$ \\
O1 & $4 b$ & $m .$. & 1 & $1 / 4$ & $0.0605(2)$ & $-0.3597(4)$ & $2.05(4)$ \\
O2 & $8 c$ & 1 & 1 & $0.4414(3)$ & $0.3625(2)$ & $-0.3672(8)$ & $2.05(4)$ \\
H1 & $4 b$ & $m .$. & 1 & $1 / 4$ & $0.1581(12)$ & $0.1911(2)$ & $2.05(4)$ \\
H2 & $8 c$ & 1 & 1 & $0.3530(12)$ & $0.3137(6)$ & $0.1934(5)$ & $2.05(4)$ \\
H3 & $8 c$ & 1 & 1 & $0.3521(14)$ & $0.2125(7)$ & $-0.2471(4)$ & $2.05(4)$ \\
H4 & $4 b$ & $m .$. & 1 & $1 / 4$ & $0.3718(14)$ & $-0.2345(5)$ & $2.05(4)$ \\
H5 & $8 c$ & 1 & 1 & $0.31782(5)$ & $0.01984(3)$ & $-0.0165(6)$ & $2.05(4)$ \\
H6 & $8 c$ & 1 & 1 & $0.51996(8)$ & $0.33460(13)$ & $-0.0331(10)$ & $2.05(4)$ \\
H7 & $8 c$ & 1 & 1 & $0.45229(8)$ & $0.43672(12)$ & $-0.0333(10)$ & $2.05(4)$ \\
\hline
\end{tabular}

Table S4. Selected atomic distances of 1.

\begin{tabular}{cc}
\hline Atoms & Distance \\
\hline $\mathrm{C}(1)-\mathrm{N}(1)$ & $1.4729(12) \AA$ \\
$\mathrm{C}(1)-\mathrm{C}(3)$ & $1.528(1) \AA$ \\
$\mathrm{C}(2)-\mathrm{N}(1)$ & $1.473(2) \AA$ \\
$\mathrm{C}(2)-\mathrm{N}(2)$ & $1.473(2) \AA$ \\
$\mathrm{C}(2)-\mathrm{C}(4)$ & $1.528(2) \AA$ \\
$\mathrm{C}(3)-\mathrm{O}(1)$ & $1.443(3) \AA$ \\
$\mathrm{C}(4)-\mathrm{O}(2)$ & $1.443(6) \AA$ \\
\hline
\end{tabular}




\section{XRPD/PDF co-refinement}

In this work, the laboratory XRPD pattern and synchrotron PDF were analysed by refining parameters common for both datasets, such as lattice parameters, translations, rotations, bond lengths, torsions and thermal displacements. Before the co-refinement can be carried out, both datasets must be weighted in order to equally contribute to the refinement. This means finding a weighting factor for XRPD pattern and/or PDF where both methods can give together the best result. In the present work, we performed refinements with different weighting factors applied to the PDF dataset. Figure 19 shows the PDF and XRPD specific $R_{w}$ values for each weighting during refinement with $C 2$ space group. The best weighting factor for our data (value $=10^{5}$ ), which was used for XRPD/PDF co-refinement (black circle), corresponds to approximately ||$_{\mathrm{Xrpd}} \mid /\left(||_{\text {pdf }} \mid 10\right)$, where $\mathrm{I}_{\mathrm{XPPD}}$ is the integrated intensity of the XRPD pattern and $I_{\text {pdf }}$ for the PDF plot)

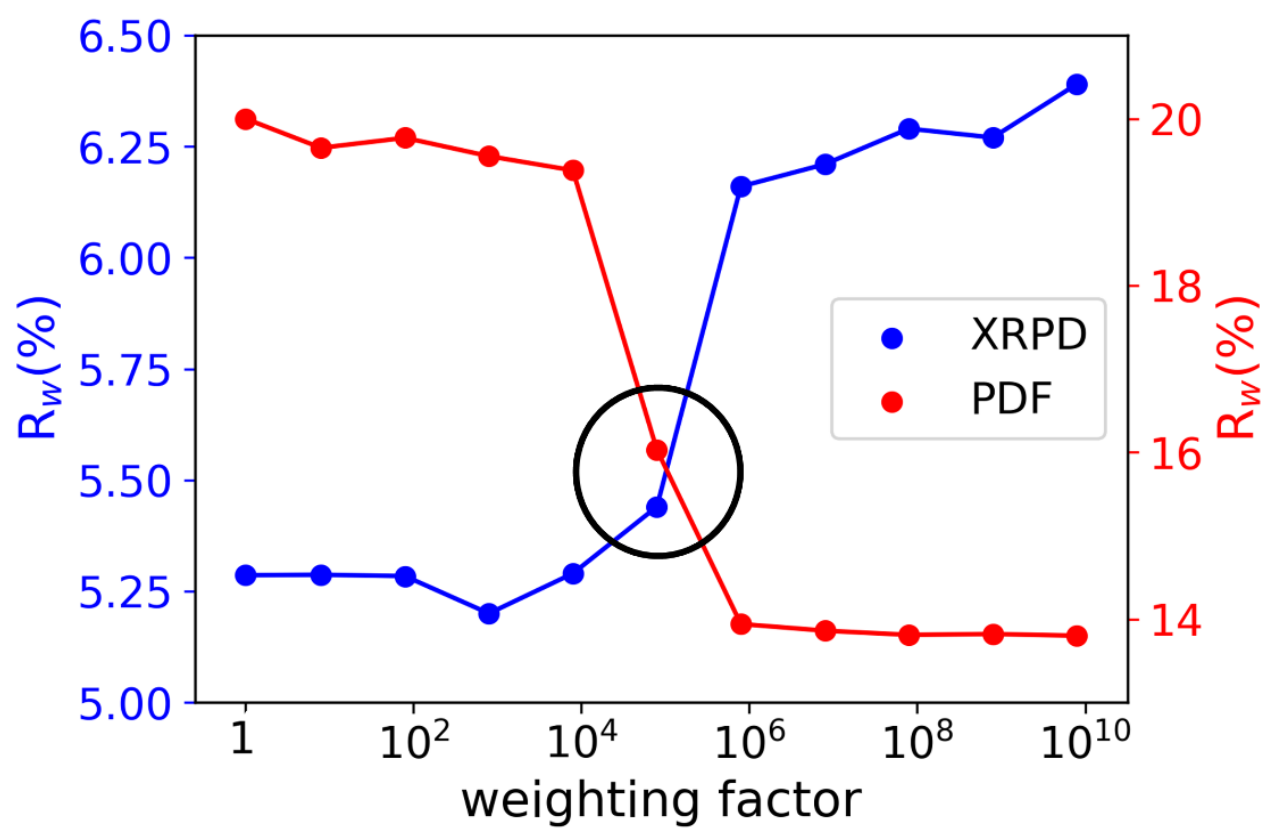

Figure S20. Weighting factor applied to PDF data and used for co-refinement versus agreement factor ( $\left.R_{w}\right)$ obtained from XRPD (blue) and PDF (red) refinement with the model in C2 space group.

\section{References}

(1) Coelho, A. A.; Chater, P. A.; Kern, A. Fast synthesis and refinement of the atomic pair distribution function. Journal of Applied Crystallography 2015, 48, 869.

(2) Juhás, P.; Farrow, Christopher L.; Yang, X.; Knox, Kevin R.; Billinge, Simon J. L. Complex modeling: a strategy and software program for combining multiple information sources to solve ill posed structure and nanostructure inverse problems. Acta Crystallographica Section A Foundations and Advances 2015, 71, 562. 